\title{
A BRIEF ANALYSIS OF THE REHABILITATION SYSTEMS OF CHILDREN WITH SPASTIC FORMS OF MOTOR DISORDERS
}

\author{
Nikolay Moga \\ Department of Orthopedics and Rehabilitation \\ National Pedagogic Dragomanov Uneversity \\ 8/14 Turgenivska str., Kyiv, Ukraine, 00154 \\ moga2003@ykr.net
}

\begin{abstract}
The article briefly analyzes the main studies in the field of overcoming spasticity within the confines of medical and partly pedagogical approaches. It was singled out the range of the main aspects that can be used as a basis for the correction of motor disorders spasmodic forms in children of early and preschool age by means of adaptive physical education from the position of pedagogy, medicine, physiology and neurology in their constituent components. The paper studies the methods of overcoming spasticity suggested by specialists of various profiles containing promising key ideas for further research. Efforts have been made to combine possibilities of medical methods of spastic motor disorders forms rehabilitation with pedagogical capabilities of adaptive physical education. Such an approach can guarantee the complexity of various forms of spastic motor disorders, which can positively affect the construction of an individualized correctional strategy and tactics in further rehabilitation work. Analyzing the literature sources, we did not find a single universal method for the complete or at least prevailing overcoming of spasticity in children and adults.
\end{abstract}

Keywords: spasticity, children of early and preschool age, motor disorders, rehabilitation methods.

\section{Introduction}

Among the priority directions, the Ukrainian educational system reformation deals with the increasing of the level of the correction work with children who have limited health possibilities and also it deals with the inclusive education for such children. In its turn, it involves the improvement of the physical rehabilitation system for disabled children. Perhaps the most numerous group today is a group of children with musculoskeletal disorders caused by various nosologies. In recent years the motor disorders of the neurogenic etiology became notably common, i. e. the disorders connected with depression or trauma of the central nervous system. Moreover, these disorders are often not even diagnosed by physicians at the early stages of babies' development. However, later these disorders become more intense and cause the dysontogenesis of the child's psychophysical development. So, there is the only one conclusion: early age has to become a moment of increased attention on the part of scientists and practitioners, since the plasticity of the nervous system during this period is the greatest and it provides exceptional opportunities for correcting existing motor disorders.

The initial interest for us is represented by the medical methods of overcoming children muscular spasticity, in future these methods can serve as a basis for their adapted usage in correctional pedagogy. The results of studies conducted by a number of authors using the method of fixed stretching of spastic muscles with the help of braces or splints are widely used in neurological clinics and rehabilitation centers [1-5]. This antispastic method is based on the fact that muscle stretching lengthens muscle receptors (spindles) and it leads to common hypertonic muscles tone decrease. Many techniques are used to control changes in muscle tone (spasticity). One of them is the determination of the angular velocity when the stretch reflex works out and the resistance to the spastic limb passive movement occurs. These approaches can be taken as a basis for the design of the physical development monitoring of children of early and preschool age with spastic motor disorders.

The effect of a 6-hour fixed stretch of spastic muscles was also studied. The author found that a decrease in spasticity of muscles leads to an increase in the joint angle of the limb. These changes in muscle tone were accompanied by a decrease in resistance to the passive movement of the spastic limb [6]. 
Interesting are studies in which the comparative characteristics of the effect of fixed stretching with the help of different langets on the spasticity of the muscles of the upper limbs were presented [5]. Two methods of using braces were employed. In one case, two-hour fixed muscle stretch was implemented, in the other - the limb muscles weren't fixed. During this process only the joint angle under control was measured, and the elbow or carpal angles of these extremities were also fixed. It was found that despite the significant change in the angles of the limb, the integrated EMG (electromyogram) of the spastic muscles enduring a fixed extension of the spastic muscles did not undergo significant changes. Initial data were obtained with non-fixed muscle stretching. The author believes that EMG is an important indicator of determining the muscle tone (spasticity) of a patient with damaged CNS. Thus, it didn't become possible to find a reliable therapeutic effect using a fixed stretch of spastic muscles.

Besides the method of the limb fixing by a brace in order to have spastic muscles stretching in the process of the spasticity treatment, the method of fixed periodic stretching using the mechanical devices is also implemented. A technique was proposed for stretching muscles using a mechanical device that spreads legs symmetrically in different directions for 30 minutes [7]. Spastic muscles were stretched in two ways:

1. Moving apart (splitting) and fixation, while maintaining the maximum angle between the legs and periodical increasing of this angle by moving the legs apart and its temporary fixing after each increase of the angle between the legs.

2. Moving the legs apart to the maximum and fixing them.

A stretching of spastic hamstrings according to the program of fixed periodic stretching was also proposed with the help of the same mechanical device fixed on the table.

Observing the high muscle tone in the lower limbs, before the beginning of the verticalization body training, a series of exercises providing relaxation for the spastic muscles in the horizontal and vertical positions must be used. A team of specialists developed a shield that consists of two parts: a vertical wall and a horizontal platform, connected by veils to change the angle of the foot support [8]. The purpose of the simulator is as follows:

1. Decreasing spasticity of the muscles, bringing the hips together and turning them inward.

2. Overcoming flexion contractures in the knee joints.

3. Overcoming the equines installation of the feet, i. e. the tip foot.

4. Stretching of the muscles and tendons of the posterior surface of the hips and legs.

5. Creating the ability to support the feet and the function of straight-standing.

6 . Forming the manual manipulative actions with objects.

Studying the features of correction of spastic forms of motor disorders, we can also pay attention to the technique of muscle tone normalizing by eliminating pathological reflexes and improving the coordination of movements according to [9]. The author identifies two types of techniques depending on their physiological orientation:

a) methods of influencing the state of the neuromuscular apparatus mainly through the structures of the segmental level of the spinal cord;

b) methods of influencing the activity of the neuromuscular apparatus, mainly directed at the structures of the brain.

At the same time, the author uses the methods of muscle tone inhibition by means of stimulating their tendons, as well as methods of "reciprocal inhibition" of muscle tone, the purpose of which is as follows [10]:

- Suppression of pathological and stimulation of normal neurological reactions;

- Decrease of pathological muscle tone;

- Stimulation and development of the reactions of "straightening" through the consistent normalization of the act of holding and moving the head, as well as stimulation and performance of the functions of the muscles of the back and pelvis;

- Prevention and correction of deformities of the limb joints.

In the complex, correction and recovery work combines several components: treatment gymnastics, manual therapy, linear-energetic and nerve-point massage. 
The well-known method of treating spasticity by means of dynamic muscle stretching has found wide application among physical therapy methodologists in neurological, neurosurgical clinics and rehabilitation centers and sanatoriums [11]. The basis of this treatment is a slow stretching of spastic muscles and holding them in this position for several seconds. After the muscle relaxes, it is slowly stretched again. This procedure is repeated successively several times. Further, the direction of movement of the limb changes, so that other spastic muscles can be included in the stretching procedure. During the session, the direction of limb movement changes several times to repeat the stretching of the entire group of spastic muscles. Technique of spastic muscles stretching requires certain skills and extraordinary attention of the physical therapy methodologist regarding each movement of the limb. The methodologist who performs the procedure of spastic muscle stretching must feel by hands the limit of the stretched muscle capabilities in order to stop stretching immediately without damaging the muscle or its tendon. The stretching of the muscle should occur with periodic pressure on the limb at a slow pace, but with increasing strength for 3040 seconds. Then the limb straightens, and after a few seconds this procedure repeats.

The effect of cyclic strains on spasticity of the lower limbs was studied. Cyclic stretching of the muscles was carried out with the help of an electro-cycloergometer, relatively rigidly fixing the feet of spasmodic legs [12]. The authors have proved that with an increase in the frequency of cyclic stretches respectively, electric reflex responses become more frequent.

Widely known in medicine is the orthopedic method, which is carried out using various braces, splints and removable joint-immobilizers. They are fixed on the limbs and passively affect the muscles of the elbow, wrist, knee, ankle joints, contributing to passive lengthening of the tendons. The essence of this method is to reduce the increased strain mainly in the lower extremities in the passive position.

In medicine, there is also a method of tenotomy (from the Greek "tenon" - tendon and "temeo" - cut): the operation associated with cutting the tendons for all kinds of contractures. This method was known even in 1811, Michaelis used it, and later Stromeyer developed it in details. In the post-Soviet space, such operations have also been practiced for many years in Tula. We will not deny certain effectiveness of this method, but we do not see the possibilities for its direct application in corrective physical education of children with spastic motor disorders. At the same time, it is possible to discuss the prospects of the tenotomy method in combination with specially adapted for the postoperative period corrective physical exercises consolidating the achieved effect against the background of strengthening functionality of ankles.

All these studies belong to the section of medicine. At the same time, there is a shortage in researches in the field of pedagogy, in particular, in the correctional physical education of children. In our opinion, the ways of transformation of purely medical approaches (physiotherapeutic, surgical, medicinal, therapeutic physical fitness, etc.) to overcome spasticity and its negative consequences in correctional methods that are acceptable in the pedagogy of early and preschool childhood are at the junction of medicine and pedagogy.

\section{The aim of research}

Proceeding from the foregoing, the purpose of this article is a preliminary search for possible ways of transforming medical methods for overcoming spasticity (and its consequences) in pedagogical approaches based on the possibilities of corrective physical education.

\section{Ways of transformation of medical methods to overcome spasticity in pedagogy}

Further on, we will elaborate on such a direction of physical education of children as diagonal gymnastics, which has many varieties depending on the corrective-improving tasks.

Proceeding from the needs of corrective physical education of children, spiral forms of muscular-fascial gymnastics deserve undoubted attention [13]. It is designed to relieve muscle tension from the spine and limbs. Its essence is as follows:

1. First a diagonal sequential stretching of the muscles is performed from the left shoulder to the right hip joint, then the same, but from the right shoulder to the left hip joint. 
2. Stretching is replaced by contraction, according to the same schema: from the left shoulder - to the right hip joint, from the right shoulder - to the left hip joint.

3. Further, there should be a stretching of the spine and muscular corset along the central axis: the sacrum - cervical part of the spine.

4. The next stage is the contraction of the spine and the muscular corset along the central axis: the sacrum - cervical part of the spine.

5. After that the eight-shaped trunk movements performed according to the next scheme follow: the movement from the left shoulder diagonally down to the area at the width of the palm below the scapula (the area of the transition of the thoracic spine to the lumbar, zone of the diaphragm) is performed alternately straining the muscles, then continuing along the diagonal - to the right hip joint, from which through the sacrum the movement leads to the left hip joint, from the joint the movement is risen diagonally upwards to the zone of the transition of the thoracic spine to the lumbar and even higher, to the right shoulder; completing the cycle, the movement goes from the right shoulder through the zone of the 7 th cervical vertebra to the left shoulder. The cycle closes.

6. The same is done in the opposite direction: from the right shoulder through the trunk - to the left hip joint, then through the sacrum - to the right hip joint, from there through the trunk - to the left humerus and further, through the area of the 7 th cervical vertebra - to the right shoulder joint. The cycle closes, conceptually forming the eight-shape movement.

7. The same diagonal, central-medial and "eight-shape" techniques are performed separately on each limb: on the left and right hands, and then on the left and right legs.

8. After working on the trunk and extremities, it is necessary to apply all the above techniques to the cervical-collar zone according to the same principle.

9. At the end of the complex of diagonal gymnastics, the muscles of the face (mimic muscles) should be worked out according to the same scheme: the diagonal - the central axis - the "eight-shape" technique.

According to the author of the method, the mentioned above gymnastics can be performed in a different way: starting with the hands and feet, then proceeding to the trunk, neck and face. Both options are equally useful and can be selected at will.

As for this approach, in our opinion, the essence is in the following aspects:

1. Muscles and fascia in this gymnastics are considered to be a single musculofascial complex, which will also have to be worked on complexly.

2. The combination of stretching and contraction (with preservation of the stretching primate) corresponds to the natural functionality of the muscles for elongation and shortening and can be used as a basis for corrective exercises in adaptive physical education of children with spastic motor disorders.

3. The diagonal, centrist and "eight-shape" approach as for working on the skeleton and muscle corset conveys the structure and functions of the musculofascial formations in the human (child) body the most accurately (harmoniously).

The disadvantages of this type of diagonal gymnastics include its complexity (almost impossibility) in terms of conscious relaxation and contraction of local muscle sites by children of early and preschool age. If to speak about the biological aspect, in the proposed variant of diagonal gymnastics we notice a certain disregard to the universal cephalo-caudal principle, which envisages the development, formation, training and rehabilitation of the musculoskeletal system in the direction "from top to bottom, from the crown to the toe". That is why, in our opinion, exercises for the facial muscles and cervical collar zone should be the first in the methodical sequence of the health correcting training.

Separately, we should dwell on diagonal gymnastics for children and adults with cerebral pathology. A complex of such gymnastics was developed in combination with the reception of amino acid components [8]. This complex in accordance with the method of diagonal curative gymnastics, in contrast to the conventional ones, presupposes an active and passive effect on specific receptors of the musculoskeletal system (muscles of the hands and feet) for the purpose of normalization through impulses in the CNS of brain structures (hypothalamus, cerebellum, reticular formation, motor area of the cortex of the brain, oblong and middle brain) and spinal cord, the 
activity of which ensures the maintenance of the correct posture; locomotion - walking, running, swimming, communication - writing, speaking; gesticulation; facial expressions; manipulation, i. e., substantive activity. As a result of the application of this technique, the equilibrium and mobility of the processes of inhibition and stimulation increase, the lost motor functions restore and the new ones develop, the motor-visceral reflex reactions are normalized.

The principles underlying the diagonal gymnastics:

1. Regularity, consistency and continuity of conduct.

2. Strict individualization.

3. Registration of the form and stage of the disease, clinical symptoms.

4. Gradually dosed increase in physical activity.

The complex consists of preparatory exercises (15-20\% of the total duration), basic exercises $(65-75 \%)$ and final exercises (10-15\%). The procedure allows:

1. To train certain muscle groups in a harmonious way.

2. To improve motor coordination and stamina.

3. To reduce the pathologically increased muscle tone.

4. To apply it to patients of any age.

5. To reduce psychoemotional stress (due to the use of specially selected rhythmic music and breathing exercises).

Diagonal gymnastics contributes to:

1. Decreased muscle tone.

2. Improved coordination and accuracy of movements.

3. Increased overall mobility.

4. Developed new skills for adequate movement.

5. Reduced hyperkinesia.

Indications and contraindications.

This technique is used as stimulating and restorative method for various diseases of cerebral genesis, accompanied by motor disorders, sensitivity disorders, vestibular disorders.

Basically the method involves simultaneous and alternate flexion-extension in the major joints of the upper and lower limbs. In this case, the cross-links must appear in the structure of the central nervous system. The cross-coordination phenomenon is very promising to our mind, because it is the basis of interhemispheric regulation of locomotion.

System of Intensive Neurophysiological Rehabilitation (SINR) appeared in Ukraine more than 20 years ago [14]. The basis of this technology is the treatment of patients with cerebral palsy, osteochondrosis, sequelae of injuries and nervous system organic damage. The rehabilitation system technique includes biomechanical correction of the spine and large joints in combination with a complex of therapeutic measures (reflexotherapy, therapeutic gymnastics, massage system, rhythmic gymnastics, mechanotherapy and apitherapy). Preliminary normalization of the spinal column in children with spastic forms of paresis with the help of special provisions and poses seems interesting to us in the correction of motor disorders by means of physical education.

Recently, in Ukraine, systematic studies of the correction of motor disorders (including spastic disorders) in preschool children have been carried out, mainly on the basis of corrective physical education [13].

In his doctoral thesis, the author outlined the main ways of correcting motor disorders of children with cerebral type of motor disorders, which are organically intertwined in the scope of studies of this article. In particular, the author proposed the following "general correction formula" for the craniocerebral type of CNS depression:

"General correction formula for cerebral type of motor disorders. Contraindications for children of conditioned cerebral group:

- Initial positions, posture and exercises with mechanical pressure on the skull (head): stand on the head, the wrestling bridge (when the child is fixed only by feet and head), all kinds of rolls which include head support (forward, backward, running, over obstacles), active head support of a partner or a subject. 
- Shock-ballistic skull (head) effects: hand and feet boxing, strikes by any (even relatively soft) objects like a ball, roller, bag with sand, slaps, flicks, strokes by the surface of support (for example, mat or carpet) in the lying-horizontally position; heading a ball or other objects, etc.

- Poses with obvious head-down positioning: apart from the stand on the head, here you can also include a rack on the shoulder blades ("candlestick"), different hangs upside down, lying on a sloping surface head down, etc.

- Exercises and types of competitions (relay race) with muscular tension: lifting and carrying heavy objects, dragging a rope, a pole or a stick; dragging or pushing a partner (from a circle), holding the weight of the partner in acrobatic positions etc.

- Exercises with fast turns in lying position, rotation on different devices or simulators.

- Sharp transitions from a lying position to a standing position.

- Hyperactive powertrain, especially performing the role of the leader of the team: during running for short distances, jumps and other relay races and competitions.

- Loud music, noise, screams, and other acoustic stimuli.

- It is also clear that when we speak about the contracted foot its front part is overloaded; for such children it is necessary to exclude standing, walking, running, dancing movements on the tips of the toes. This position of the ankle is the most undesirable, as well as the turning of points of the feet inside.

\section{Results of the research}

Recommended correction strategy:

1. Relaxation regime in the psychophysical activity of the child - involves the use of techniques that reduce the neuromuscular and mental excitement of hyperactive disinhibited children:

A) Gravitational skeleton (spine) relief in horizontally lying positions (in a fetal position on the side, on the back, on the abdomen, in the posture of a half-embryo on one of the parents, on a big ball, on a roller, etc.). This approach automatically reduces gravity stimulation of the skeleton, which leads to a natural decrease in the neuromuscular tone, which in turn reduces the degree of irradiation (spread) of excitation in the cerebral cortex, which makes the child calmer.

B) Muscle relaxation itself involving the systematic usage of methodical techniques that will reduce the hypertonicity in specific muscle groups, such as in the upper or lower limbs.

2. Working on toughness of joints (passively and actively) of the upper and lower limbs.

3. Overcoming of muscles-antagonists paresis (strengthening of muscles-extensors of the trunk and extremities). In other words, if at the test a child or a group of children were mentioned to have pathological tension of the flexor muscles - contrariwise stimulation, strengthening and training of extensor muscles of the upper extremities in particular should be encouraged.

4. Improvement of the abilities of the hands - refers to the hands, radial joints, which are in a bent and compressed position. The above-mentioned support-rectifying exercises will improve the function of the hands support, without which the complete development of a child and effective maturation of cranial structures are impossible. G. Doman believes that the function of crawling for a child is extremely important and it stimulates the formation of the base of the brain, the conventional first three levels of seven. Hand and carpal movements play an important role in the child's linguistic development, stimulating intellectual manifestations of children. For the purpose of unfolding a hand of a child of a conditional cerebral group and for making it fully functional and improving its reference capacity, for many years in the author pedagogical Center we have been using so-called "treads": these are compact devices for capturing them by hand or for producing hand support, which have different shapes (configuration) and stimulate the abilities of the hands: it is necessary to make thrust to spot in lying position or to crawl fixing them by hands.

5. Overcoming the wrong sets in the limbs and trunk provides the following correction directions:

A) With predominant bending set in the extremities, it is necessary to apply corrective exercises aimed at their extension and straightening; to encourage the children to repulse or hold the posture with straightened limbs, boxing with nominal punching bag. 
B) Typical pulling of shoulders and hips together is overcome by exercises with moving of arms and legs to the sides.

C) To strive for the supination of arms and legs which means the deployment of hands and feet from the outside.

D) Bending the trunk in the zone of the hip joints, to make it lean back stretching spasmodic iliac-lumbar muscles and strengthening the sciatic muscle mass.

6. Improvement of leg support is supposed to overcome such malfunctions in the lower limbs, as the hollow foot and tip foot, which will allow to increase the foot support surface and, accordingly, the bearing capacity greatly.

7. Improvement of the functions of motion management, which is intended to focus primarily on the development of such children's static and dynamic equilibrium, adequate antigravity and vestibulomotor reactions, global coordination between the torso and limbs, orientation in small and large space, a sense of "own body scheme", fine motor skills of hands and fingers.

8. At the second stage of the corrective work, it is necessary to begin to form independent, meaningful, purposeful movements realized in certain plot-game actions.

9. Increase of the effectiveness of the main movements, especially when they are weakly formed (lying, crawling, sitting, standing, walking).

10. Stimulation of such functional systems of the body of children as cardiovascular, respiratory, thermoregulatory, digestive, excretory, etc. [6].

The above mentioned correction strategy, of course, is very complex and systematized. And though it is designed for pre-school children, it can be taken as a basis for the development of physical rehabilitation systems for young children with spastic paresis. Certain adjustments will be needed regarding the method of correction of the listed types of motor disorders, especially concerning methodical techniques of physical rehabilitation of young children.

It should also be noted that in the presented "general correction formula", the author outlined in general the problem of reducing muscle tone, which is the fundamental action in overcoming muscle spasm. In our opinion, it is necessary to pay more attention to the issues of reducing spasticity, overcoming muscle hypertonicity, including both medical rehabilitation techniques and the possibilities of adaptive physical education, combining their developing and healing potential.

\section{Conclusions}

Summarizing the contents of the article, let us draw some preliminary conclusions:

1. Problems of overcoming spasticity have been a subject of close attention of experts for quite a long time. Dominant researches in this area have been mostly carried out within the framework of medical approaches.

2. The attempts to combine the possibilities of medical methods of treating spasticity with the pedagogical capabilities of adaptive physical education were made.

3. Today there is no integrated, universal, reliable method of complete or at least prevailing overcoming of spasticity of children and adults.

The current situation determines the relevance and prospects of the research of the role of passive and active physical exercises, modes of muscle exercises, material and technical support in adaptive physical education of children of early and preschool age with spastic forms of motor disorders in their optimal combination with medical methods of treating spasticity.

\section{References}

[1] Bishop, B. (1977). Spasticity: Its Physiology and Management: Part I. Neurophysiology of Spasticity: Classical Concepts. Physical Therapy, 57 (4), 371-376. doi: 10.1093/ptj/57.4.371

[2] Bishop, B. (1977). Spasticity: Its Physiology and Management: Part II. Neurophysiology of Spasticity: Current Concepts. Physical Therapy, 57 (4), 377-384. doi: 10.1093/ptj/57.4.377

[3] Bishop, B. (1977). Spasticity: Its Physiology and Management: Part III. Identifying and Assessing the Mechanisms Underlying Spasticity. Physical Therapy, 57 (4), 385-395. doi: 10.1093/ptj/57.4.385

[4] Bishop, B. (1977). Spasticity: Its Physiology and Management: Part IV. Current and Projected Treatment Procedures for Spasticity. Physical Therapy, 57 (4), 396-401. doi: 10.1093/ptj/57.4.396 
[5] Mills, V. M. (1984). Electromyographic Results of Inhibitory Splinting. Physical Therapy, 64 (2), 190-193. doi: 10.1093/ptj/64.2.190

[6] Snook, J. H. (1979). Spasticity reduction splint. American Journal of Occupational Therapy, $33(10), 648-651$.

[7] Odeen, I. (1981). Reduction of muscular hypertonus by long-term muscle stretch. Scandinavian Journal of Rehabilitation Medicine, 13, 93-99.

[8] Hohlov, A. P., Tarakanov, O. P., Docenko, A. N. (1996). Diagonal'naia gimnastika dlia detei i vzroslyh s cerebral'noi patologiei. Moscow: Centr Sovremennoi mediciny "Primavera medika", 16.

[9] Smolyaninov, A. G., Shargorodskiy, V. S. (1996). Kineziterapiya detskogo tserebral'nogo paralicha. Kiyv, 59.

[10] Tekoryus, V. V. (1987). Korrektsiya dvigatel'nykh narusheniy pri detskikh tserebral'nykh paralichakh. Moscow: Meditsina, 19.

[11] Burke, D., Lance, J. W. (1973). Studies of reflex effects of primary and secondary spinal endings in spasticity. New Developments in electromyography and clinic Neurophysiology, 3, 475-495. doi: $10.1159 / 000394162$

[12] Diagonal'no-fastsial'naya gimnastika. Available at: http://www.osteodoc.ru/dfg.htm

[13] Efimenko, M. M. (2014). Osnovi korekciino spriamovanogo fizichnogo vihovannia ditei z porushenniami oporno-ruhovogo aparatu. Kyiv, 441.

[14] Koziavkin, V. I. (2008). Rannia reabilitaciia ditei z perinatal'nimi urazhenniami nervovoy sistemi za metodom profesora V. I. Koziavkina. Ser. social'no-pedagogichna, Kamyanets-Podilsky, $10,112-115$. 